\title{
A SIGNIFICANT POPULATION OF RED, NEAR-INFRARED-SELECTED HIGH-REDSHIFT GALAXIES
}

\author{
Marijn Franx, ${ }^{2}$ Ivo Labbé, ${ }^{2}$ Gregory Rudnick, ${ }^{3}$ Pieter G. van Dokkum, ${ }^{4}$ Emanuele Daddi, ${ }^{5}$ \\ Natascha M. Förster Schreiber, ${ }^{2}$ Alan Moorwood, ${ }^{5}$ Hans-Walter Rix, ${ }^{6}$ Huub Röttgering, ${ }^{2}$ \\ ArJen van De Wel, ${ }^{2}$ Paul van Der Werf, ${ }^{2}$ ANd Lottie VAN Starkenburg ${ }^{2}$ \\ Received 2002 December 13; accepted 2003 February 26; published 2003 March 18
}

\begin{abstract}
We use very deep near-infrared photometry of the Hubble Deep Field-South taken with ISAAC on the Very Large Telescope to identify a population of high-redshift galaxies with rest-frame optical colors similar to those of nearby galaxies. The galaxies are chosen by their infrared colors $J_{s}-K_{s}>2.3$, aimed at selecting galaxies with redshifts above 2 . When applied to our data set, we find 14 galaxies with $K_{s}<22.5$, corresponding to a surface density of $3 \pm 0.8 \operatorname{arcmin}^{-2}$. The photometric redshifts all lie above 1.9, with a median of 2.6 and an rms of 0.7. The spectral energy distributions of these galaxies show a wide range. One is very blue in the rest-frame UV and satisfies the normal Lyman break criteria for high-redshift, star-forming galaxies. Others are quite red throughout the observed spectral range and are extremely faint in the optical, with a median $V=26.6$. Hence, these galaxies would not be included in photometric samples based on optical ground-based data, and spectroscopic follow-up is difficult. The spectral energy distributions often show a prominent break, identified as the Balmer break or the $4000 \AA$ break. The median age is $1 \mathrm{Gyr}$ when fitted with a constant star formation model with dust or $0.7 \mathrm{Gyr}$ when fitted with a single burst model. Although significantly younger ages cannot be excluded when a larger range of models is allowed, the results indicate that these galaxies are among the oldest at these redshifts. The volume density to $K_{s}=22.5$ is half that of Lyman break galaxies at $z \approx 3$. Since the mass-to-light ratios of the red galaxies are likely to be higher, the stellar mass density is inferred to be comparable to that of Lyman break galaxies. These red galaxies may be the descendants of galaxies that started to form stars at very high redshifts, and they may evolve into the most massive galaxies at low redshift.
\end{abstract}

Subject headings: galaxies: evolution — galaxies: high-redshift

\section{INTRODUCTION}

One of the most effective ways to find high-redshift galaxies is the Lyman break selection technique (Steidel \& Hamilton 1993; Steidel et al. 1996a, 1996b). This selection method relies on the strong break in the rest-frame far-UV spectrum of strongly star-forming galaxies. The density of bright Lyman break galaxies at $z \approx 3$ is comparable to that of $L_{*}$ galaxies nearby, and this population is a major constituent of the highredshift universe. Other selection techniques based on submillimeter or X-ray emission have yielded complementary samples, with generally little overlap, and much smaller number densities (e.g., Smail et al. 2002a; Barger et al. 2002). The submillimeter galaxies may, however, contribute significantly to the overall star formation rate.

All of these techniques select galaxies that are very different from normal galaxies in the present-day universe, the light of which is dominated by rather evolved stars. Normal galaxies are too large and too faint in the far-UV to be selected as Lyman break galaxies at $z=3$ (e.g., Giavalisco et al. 1996). This raises the question of whether or not we may still be missing a significant population of galaxies at high redshift.

This issue can be addressed by means of very deep near-

\footnotetext{
' Based on service mode observations collected at the European Southern Observatory, Paranal, Chile (ESO programme 164.O-0612).

${ }^{2}$ Leiden Observatory, P.O. Box 9513, J. H. Oort Building, Niels Bohrweg 2, NL-2300 RA, Leiden, Netherlands.

${ }^{3}$ Max-Plank-Institut für Astrophysik, P.O. Box 1317, Karl-SchwarzschildStrasse 1, D-85741 Garching, Germany.

${ }^{4}$ California Institute of Technology, MS 105-24, Pasadena, CA 91125.

${ }^{5}$ European Southern Observatory, Karl-Schwarzschild-Strasse 2, D-85748 Garching, Germany.

${ }^{6}$ Max-Plank-Institut für Astronomie, Königstuhl 17, D-69117 Heidelberg, Germany.
}

infrared (NIR) imaging. A normal $L_{*}$ galaxy would have a $K$ band magnitude of $\approx 23$ when redshifted to $z=3$, out of reach of $4 \mathrm{~m}$ class telescopes. The development of large telescopes with good image quality and large NIR detectors has made it possible to achieve the depth necessary to select high-redshift galaxies by their observed infrared light.

In this Letter, we present results obtained at the Very Large Telescope on the Hubble Deep Field-South (HDF-S; Williams et al. 2000; Casertano et al. 2000), as part of the Faint Infrared Extragalactic Survey (Franx et al. 2000). We imaged the WFPC2 field of the HDF-S with ISAAC in three passbands $\left(J_{s}, H\right.$, and $K_{s}$ ), with a total integration time of $103 \mathrm{hr}$. The data processing and results are discussed in Labbé et al. (2003). The first results based on a subset of the data were presented by Rudnick et al. (2001). All magnitudes given here are in the Johnson system unless noted otherwise.

\section{INFRARED SELECTION OF HIGH-REDSHIFT GALAXIES}

We consider the selection of galaxies that are not dominated by an unobscured starburst in their rest-frame UV-optical light. We wish to define a simple photometric criterion that can select such galaxies, similar to the $U$-dropout criterion to select Lyman break galaxies. Unfortunately, the rest-frame optical spectra of optically red galaxies do not contain any feature as prominent as the Lyman break of star-forming galaxies. However, we can use the Balmer break and the $4000 \AA$ break to select high-redshift galaxies. The lower strength of this feature implies that we need to obtain very deep photometry. Since the breaks shift into the $J_{s}$ band at $z \approx 2$, a red $J_{s}-K_{s}$ color is a simple and effective criterion. This is illustrated in Figure 1, where we show tracks of $J_{s}-K_{s}$ color versus redshift for model spectra taken from Bruzual \& Charlot (1993; G. Bruzual \& S. 


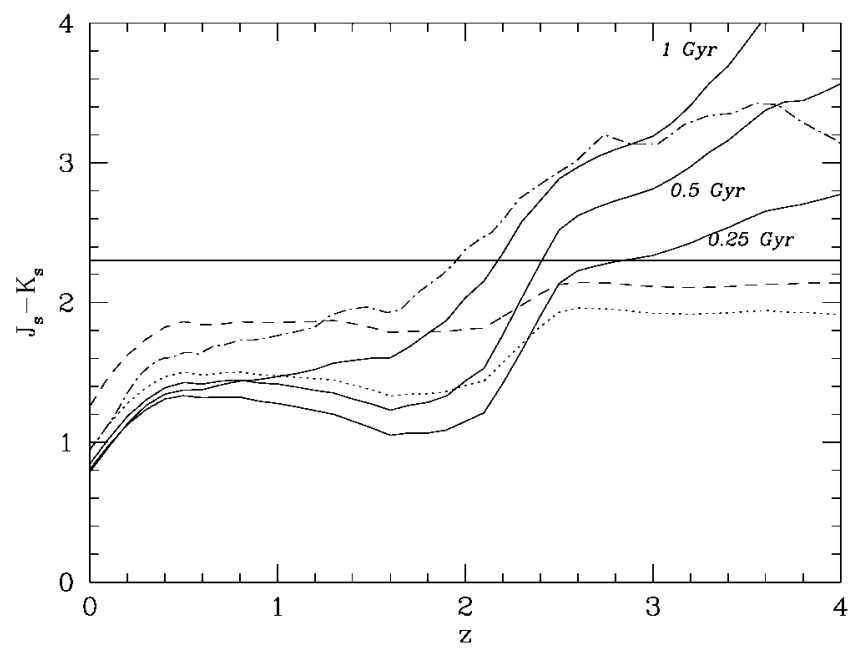

FIG. 1. $-J_{s}-K_{s}$ color as a function of redshift for several galaxy spectra. The solid curves indicate single-age stellar populations with ages of $0.25,0.5$, and $1 \mathrm{Gyr}$. The colors exceed $J_{s}-K_{s}=2.3$ only for $z>2$ as a result of the Balmer break/4000 $\AA$ break moving into the $J_{s}$ band. The dotted and dashed curves indicate models with continuous star formation with ages and reddenings of $1 \mathrm{Gyr}, E(B-V)=0.15$ and $100 \mathrm{Myr}, E(B-V)=0.5$, respectively. Many galaxies with continuous star formation will not reach $J_{s}-K_{s}=2.3$, unless they are even older or have larger reddening. The dash-dotted curve indicates the color evolution of a single-burst population that formed at $z=$ 5 , and it also satisfies the color criterion above $z=2$.

Charlot 1996, private communication). If we impose a criterion $J_{s}-K_{s}>2.3$, we select galaxies with $z>2$, even if we allow for dust extinction.

Exceptions will occur, obviously. Some of the lower redshift, extremely red objects have $J-K>2.3$ (e.g., Smail et al. 2002b), but their surface density is low $\left(\approx 0.2 \mathrm{arcmin}^{-2}\right)$. Hall et al. (2001) selected four galaxies with $J-K>2.5$ and found photometric redshifts $z \geq 2$, in agreement with our simple tracks.

Figure 2 shows the $J_{s}-K_{s}$ versus $I-K_{s}$ colors of the galaxies in the HDF-S with $K_{s}<22.5$. We find 14 galaxies down to this limit with $J_{s}-K_{s}>2.3$. Since our effective area is $4.7 \mathrm{arcmin}^{2}$, this implies a surface density of $3 \pm 0.8$ galaxies $\operatorname{arcmin}^{-2}$, which is 7 times that of bright Lyman break galaxies around $z=3$ selected by the ground-based $U$-dropout technique (Steidel et al. 1996b). The redshift distributions of the two samples are significantly different, however, and the volume densities are more comparable as we shall see below.

The full spectral energy distributions (SEDs) of a subset of the galaxies are shown in Figure 3. The majority of the galaxies show a distinct break in the NIR, which we interpret as the Balmer/4000 ̊ break. The galaxies have a wide range in optical-NIR colors (Figs. 2 and 3). We find five extremely red galaxies with $J_{s}-K_{s}>3$, and four of these are red in all colors. Their colors are rather similar to the galaxies found by Dickinson et al. (2000) and Im et al. (2002).

\section{REDSHIFTS AND DENSITY}

Most of the galaxies are extremely faint in the optical, with a median $V$-band magnitude of 26.6. As a result, it is very difficult to measure redshifts spectroscopically with current instrumentation, and we have to rely on photometric redshifts instead. Fortunately, the spectral breaks that many of the galaxies show in the NIR help us to derive the photometric redshifts.

We have used the photometric redshifts published by Labbé et al. (2003), based on an algorithm developed by Rudnick et al. (2001; G. Rudnick et al. 2003, in preparation). The SED is

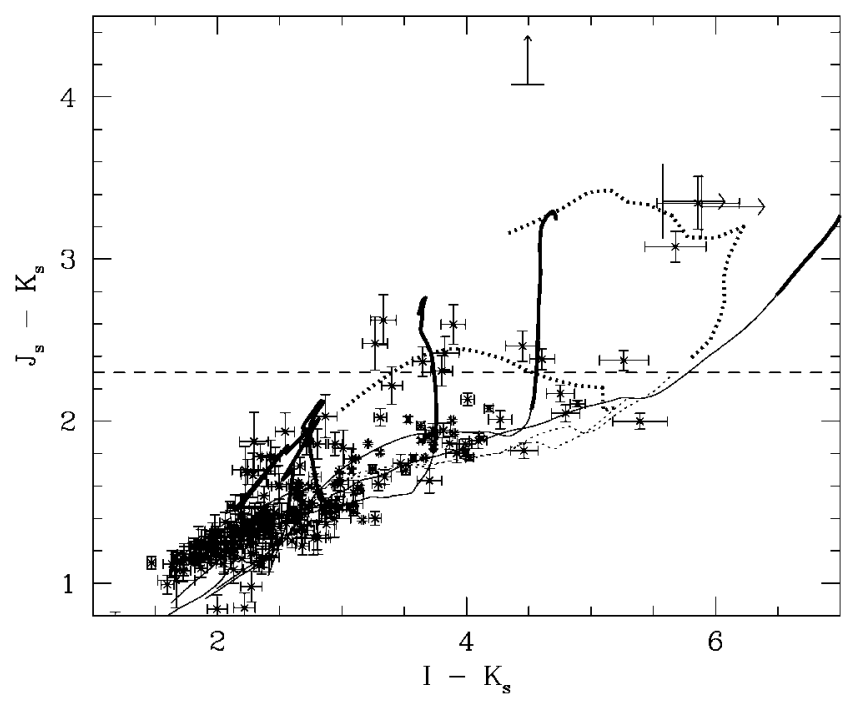

FIG. 2. $-J_{s}-K_{\mathrm{s}}$ colors vs. $I-K_{\mathrm{s}}$ colors of $K$-band-selected galaxies in the HDF-S. A significant population of galaxies with $J_{s}-K_{s}>2.3$ is present, with a large range in $I-K_{s}$ colors. The solid curves indicate color tracks obtained by redshifting the galaxy spectra measured by Coleman, Wu, \& Weedman (1980). The dotted curves indicate single-age stellar populations with formation redshifts of 5 and 3 . The curves are drawn thick for $2<z<4$.

fitted with a linear combination of templates based on observed spectra of nearby galaxies. It gives good agreement with the spectroscopic redshifts available in the HDF-S, with an average $|\Delta z| /(1+z)=0.08$. We verified that the derived density remains the same within the errors if another method is used (the HYPERZ code by Bolzonella, Miralles, \& Pelló 2000). Spectroscopic confirmation of the photometric redshifts is urgently needed obviously.

The photometric redshifts of the red sample with $K_{s}<$ 22.5 range from 1.92 to 4.26 , with a median of 2.6 and an rms of 0.7 . The volume density is derived in the interval $2<z<$ 3.5 , identical to that of $U$-dropouts selected from WFPC 2 imaging. Eleven galaxies lie in this redshift range, resulting in a volume density of $0.0014 \pm 0.0004 h^{3} \mathrm{Mpc}^{-3}$. Errors in the photometric redshifts are likely to reduce the measured volume

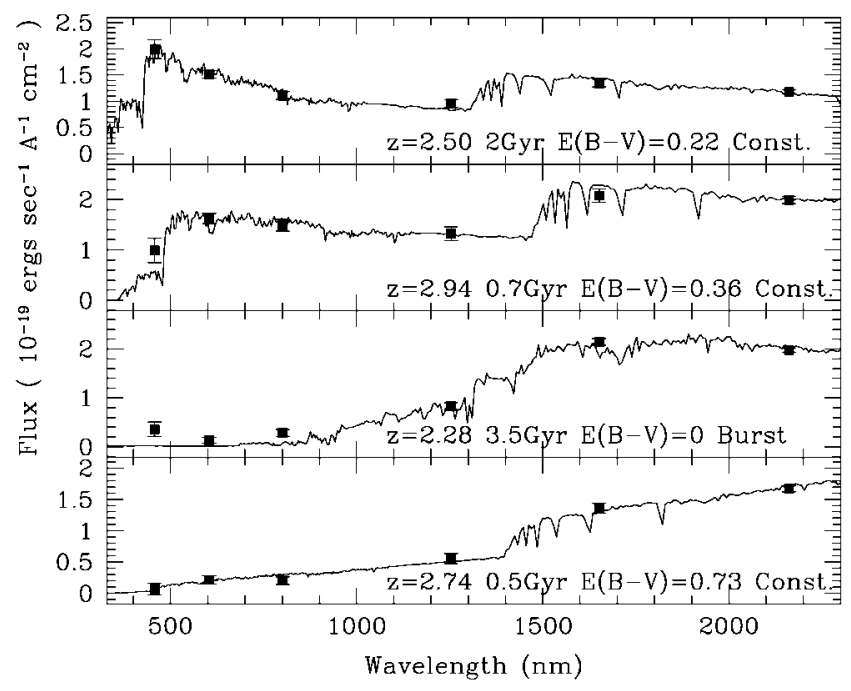

FIG. 3.-SEDs of four galaxies with $J_{s}-K_{s}>2.3$. They span the full range in $I-K_{s}$ color. All the galaxies show a break between the $J_{s}$ and $K_{s}$ bands. The curves show stellar population fits with either constant formation and reddening or unreddened single-age bursts. 
density since they will push galaxies outside the sampled volume. The derived volume density is half that of Lyman break galaxies at $z \approx 3$ to $R_{\mathrm{AB}}=25.5$ (Steidel et al. 1999). Hence, the contribution of this sample to the overall density is substantial. The variations due to large-scale structure are the main source of uncertainty. We note, for example, that the number of galaxies with red $I-H$ color is much higher in the HDF-S than in the Hubble Deep Field-North (Labbé et al. 2003). On the other hand, our second deep field of $5^{\prime} \times 5^{\prime}$ contains a similar surface density of $J_{s}-K_{s}$ selected galaxies as HDF-S to $K_{s}=21$ (van Dokkum et al. 2003). Surveys over larger areas are needed to establish how typical these areas are, and they are now in progress.

\section{COMPARISON WITH LYMAN BREAK-SELECTED SAMPLES}

The large volume density derived above raises the question of how the red galaxies are related to the "classical" Lyman break galaxies (Steidel et al. 1996a, 1996b). In principle, a galaxy can be both red in $J_{s}-K_{s}$ and a Lyman break. In order to compare the two samples, we select galaxies in the HDF-S with $2<z<3.5$ and $K_{s}<22.5$, and we apply the criteria for $U$-dropouts as defined by Steidel et al. (1996a). Surprisingly, the two samples are nearly disjoint. Only one galaxy satisfies both criteria. Many of the $J_{s}-K_{s}$ galaxies fail to satisfy the $U$ dropout criteria because they are too faint in the $B$ or $V$ band. Even with HDF-S quality data, it is not possible to establish whether or not they have a prominent break between $U$ and $B$. Conversely, the median $J_{s}-K_{s}$ color of the $U$-dropout galaxies is very blue: we find a median of 1.6 , identical to that found by Shapley et al. (2001) for a ground-based selected sample.

The disjoint nature of the two populations is illustrated in Figure 4 , where we show the $R_{\mathrm{AB}}-K_{s}$ color vs. $K_{s}$ magnitude. The synthetic $R_{\mathrm{AB}}$ magnitude was derived by averaging the $V_{\mathrm{AB}}$ and $I_{\mathrm{AB}}$ magnitudes. Galaxies of different types are indicated by different symbols. The $U$-dropout galaxies are blue and follow a well-defined color-magnitude relation, as found earlier by Papovich, Dickinson, \& Ferguson (2001). The $J_{s}-K_{s}$ selected galaxies show a very large range in color, $4 \leq R_{\mathrm{AB}}-$ $K_{s} \leq 7$.

The relative contributions of the two types of galaxies to the total observed $K_{s}$-band light is similar to their relative number densities: the galaxies with $J_{s}-K_{s}>2.3$ contribute $24 \%$ to the total observed $K_{s}$-band light from galaxies with $2<z<3.5$ and $K_{s}<22.5$, and the Lyman break galaxies contribute $55 \%$. The relative contribution of the $J_{s}-K_{s}$ selected galaxies to the stellar mass is expected to be higher since the red colors indicate higher mass-to-light ratios. Figure 4 shows the track of constant stellar mass for a single stellar population observed at $z=$ 3.0 for ages between 0.1 and 2 Gyr, based on models by Bruzual \& Charlot (1993; G. Bruzual \& S. Charlot 1996, private communication). If we use this track to correct the relative luminosities to relative masses, we derive that the $J_{s}-K_{s}$ selected galaxies contribute $43 \%$ to the stellar mass. This fraction increases further if the sample were mass-selected. Hence, the population of red galaxies is a significant component at the high-mass end.

Figure 4 can also be used to analyze the selection effects of most of the ground-based spectroscopic surveys. Usually, spectroscopic samples of $U$-dropout galaxies are selected to have $R_{\mathrm{AB}}<25.5$, indicated by the solid line. All but two of the $J_{s}-K_{s}$ galaxies have $R_{\mathrm{AB}}>25.5$ and hence most would be excluded. The $R$-band selection criterion is effective in selecting galaxies with strong, unobscured star formation but misses a

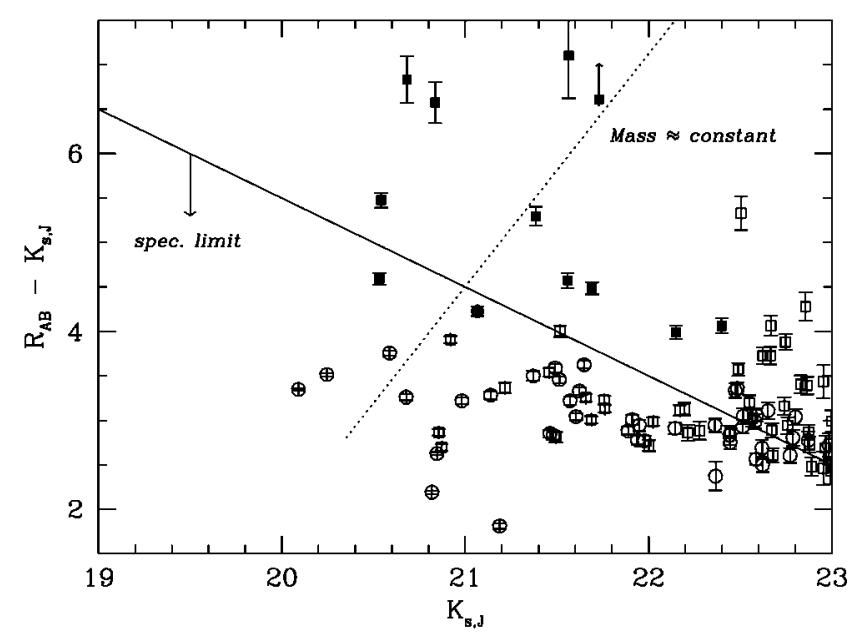

FIG. 4. $-R_{\mathrm{AB}}-K_{s}$ color vs. $K_{s}$ magnitude for galaxies with $2<z<3.5$. The $J_{s}-K_{s}$ red galaxies are indicated by filled symbols. Lyman break galaxies are indicated by the circles. The solid line indicates the magnitude limit used for most ground-based spectroscopic studies of Lyman break galaxies of $R_{\mathrm{AB}}=$ 25.5. Most $J_{s}-K_{s}$ red galaxies are missed when this magnitude limit is applied. The dotted line is a track of constant mass for a model with a single-age population. As can be seen, the $J_{s}-K_{s}$ red galaxies are slightly less luminous in the $K_{s}$ band than the brightest Lyman break galaxies in the HDF-S, but their masses may well be comparable or higher.

significant population of galaxies with redder colors. Lines of constant mass run nearly perpendicular to the $R$-band selection criterion: if we select galaxies in the optical, we miss red galaxies over a wide range of masses and up to the highest masses. Follow-up studies of NIR-selected samples are necessary to obtain a full census of the high-redshift universe; unfortunately, such studies are very hard.

\section{STELLAR POPULATIONS}

The $J_{s}-K_{s}$ selected galaxies have much redder colors than most Lyman break galaxies; this can result from older ages, more reddening, or a combination of effects. Furthermore, emission lines can contribute to some of the passbands and influence the colors. Here we attempt to understand the stellar populations of the galaxies better by analyzing the colors and SEDs. For the moment, we ignore the effects of emission lines since they are likely small (e.g., Shapley et al. 2001; van Dokkum et al. 2003; P. G. van Dokkum et al. 2003, in preparation).

The breaks in the SEDs of many of the galaxies can be used to put lower limits on the ages. Color-color diagrams using the $I-J_{s}, J_{s}-H$, and $H-K_{s}$ colors show that nine out of 14 galaxies have breaks that cannot be explained by reddened models of stellar populations with constant star formation and young ages ( $\leq 100 \mathrm{Myr}$ ). Generally, models with ages greater than $300 \mathrm{Myr}$ and higher can produce strong enough breaks to explain the features, in combination with dust.

We can use the full SED to fit models of stellar populations. We used models by Bruzual \& Charlot (1993; G. Bruzual \& S. Charlot 1996, private communication), with a Calzetti et al. (2000) reddening law. As is well known, it is very hard to disentangle the effects of age and dust in such fits (e.g., Papovich et al. 2001; Shapley et al. 2001). However, 11 out of 14 of the galaxies are badly fitted by models with very young populations (with ages $\leq 100 \mathrm{Myr}$ ) and very large reddening. The $\chi^{2}$-test rules out these fits at the $95 \%$ confidence level. Unreddened single-age bursts can be ruled out in the same way for 12 galaxies. Models with constant star formation produce a median age of 
$1 \mathrm{Gyr}$ and a median reddening of $E(B-V)=0.5$. Both the age and the reddening are about 3 times higher than the median values for Lyman break galaxies at $z=3,0.3 \mathrm{Gyr}$ and 0.16, respectively (Shapley et al. 2001). Two of the very red galaxies are best fitted by single-burst models without dust, as determined from the $\chi^{2}$. These models have an age of $3.5 \mathrm{Gyr}$. If all galaxies are fitted with a single-age population, the median age derived is 0.7 Gyr, with a large spread.

As shown by Papovich et al. (2001) and Shapley et al. (2001), the derived ages and reddening can vary by a factor of 2 if other star formation histories, initial mass functions, or reddening laws are used. The relative ages of galaxies are more stable, however. Our models are identical to those used by Shapley et al (2001); hence, the age comparison should be more secure. Again, followup studies are required to constrain the stellar populations better. NIR spectroscopy will be essential to estimate the star formation rates and reddening from emission lines.

\section{DISCUSSION}

The results presented here raise many questions. Obviously, follow-up work is required to verify how typical the HDF-S is. Deep NIR imaging over a wide area is needed to obtain a robust estimate of the surface density. Furthermore, it is critical to determine the spectroscopic redshifts of at least some of these sources. The first spectroscopic confirmation of galaxies selected in this way is presented in a companion paper (van Dokkum et al. 2003). The most pressing question of all, however, concerns the nature, formation, and evolution of these red galaxies and their relation to other high-redshift galaxies, especially to the well-studied Lyman break galaxies.

The simplest explanation would be that the red galaxies are directly related to Lyman break galaxies. They may be viewed along dusty lines of sight or during intermittent epochs of low star formation. If such a direct relation would hold, one would expect that both samples have a similar distribution on the sky, similar sizes, and similar clustering. The first results by Daddi et al. (2003) imply that the clustering of high-redshift galaxies increases rapidly with redder $J_{s}-K_{s}$ colors. If confirmed by spectroscopy and larger area surveys, it would be inconsistent with this very simple scenario.

Alternatively, the red galaxies could be a population distinct from the $z \approx 3$ Lyman break galaxies. They may have been Lyman break galaxies at higher redshifts, and they may have become redder because of a decline in the star formation, an increase in age, and an increase of metallicity (and thereby dust). All three factors are expected to play a role in realistic galaxy evolution models. In this context, it is interesting to note that models of observed Lyman break galaxies indicate that such red descendants must exist since the ages of the stellar populations are generally much younger than the age of the universe at the redshift of the galaxies (e.g., Papovich et al. 2001; Ferguson, Dickinson, \& Papovich 2002). Unless these galaxies evolve in unexpected ways, one must see older and relatively red "remnants."

In the nearby universe, the reddest galaxies are generally the most massive, with the highest ages, metallicity, and correlation length. It is possible that such a relation already existed in the early universe. The red galaxies found here would be the descendants of galaxies that started to form stars very early, and they would be related to more massive halos than those of the young Lyman break galaxies at $z=3$. In this case, their spatial densities are expected to be higher and their correlation length to be enhanced, as found by Daddi et al. (2003). Work is in progress to test these predictions further. Unfortunately, the physical processes that determine the appearance of high-redshift galaxies are not well understood; e.g., why are many strongly star-forming galaxies at $z=3 \mathrm{UV}$-bright and most counterparts at low-redshift strongly reddened? In the absence of a good understanding of the high-redshift galaxies and of the feedback processes that may regulate star formation, it is not clear whether or not a simple extrapolation of the phenomena at low redshift to high redshift is valid.

Finally, caution is required, and we should leave open the possibility that we do not understand the nature of these sources yet. We note that similar objects have been found by others (e.g., Dickinson et al. 2000; Hall et al. 2001; Totani et al. 2001; Im et al. 2002). Although we have obtained spectroscopy for a limited sample (van Dokkum et al. 2003), this is only feasible for a small fraction of the sample at this moment. NIR spectroscopy is very urgently needed to confirm that the colors that are observed are not dominated by emission lines, although it appears quite unlikely that many galaxies would be affected greatly.

The comments of the referee helped to improve this Letter. We thank the staff at ESO for their hard work in taking these data and making them available. This research was supported by grants from the Netherlands Foundation for Research (NWO), the Leids Kerkhoven-Bosscha Fonds, a NASA SIRTF fellowship, and the Lorentz Center.

\section{REFERENCES}

Barger, A. J., Cowie, L. L., Brandt, W. N., Capak, P., Garmire, G. P., Hornschemeier, A. E., Steffen, A. T., \& Wehner, E. H. 2002, AJ, 124, 1839 Bolzonella, M., Miralles, J.-M., \& Pelló, R. 2000, A\&A, 363, 476

Bruzual A., G., \& Charlot, S. 1993, ApJ, 405, 538

Calzetti, D., Armus, L., Bohlin, R. C., Kinney, A. L., Koornneef, J., \& StorchiBergmann, T. 2000, ApJ, 533, 682

Casertano, S., et al. 2000, AJ, 120, 2747

Coleman, G. D., Wu, \& C.-C., \& Weedman, D. W. 1980, ApJS, 43, 393

Daddi, E., et al. 2003, ApJ, in press (astro-ph/0303165)

Dickinson, M., et al. 2000, ApJ, 531, 624

Ferguson, H. C., Dickinson, M., \& Papovich, C. 2002, ApJ, 569, L65

Franx, M., et al. 2000, Messenger, 99, 20

Giavalisco, M., Livio, M., Bohlin, R. C., Macchetto, F. D., \& Stecher, T. P. 1996, AJ, 112, 369

Hall, P. B., et al. 2001, AJ, 121, 1840

Im, M., Yamada, T., Tanaka, I., \& Kajisawa, M. 2002, ApJ, 578, L19

Labbé, I., et al. 2003, AJ, 125, 1107

Papovich, Dickinson, M., \& Ferguson, H. C. 2001, ApJ, 559, 620
Rudnick, G., et al. 2001, AJ, 122, 2205

Shapley, A. E., Steidel, C. C., Adelberger, K. L., Dickinson, M., Giavaliscon, M., \& Pettini, M. 2001, ApJ, 562, 95

Smail, I., Ivison, R. J., Blain, A. W., \& Kneib, J.-P. 2002a, MNRAS, 331, 495

Smail, I., Owen, F. N., Morrison, G. E., Keel, W. C., Ivison, R. J., \& Ledlow, M. J. 2002b, ApJ, 581, 844

Steidel, C. C., Adelberger, K. L., Giavalisco, M., Dickinson, M., \& Pettini, M. 1999, ApJ, 519, 1

Steidel, C. C., Giavalisco, M., Dickinson, M., \& Adelberger, K. L. 1996a, AJ, 112,352

Steidel, C. C., Giavalisco, M., Pettini, M., Dickinson, M., \& Adelberger, K. L. 1996b, ApJ, 462, L17

Steidel, C.C., \& Hamilton, D. 1993, AJ, 105, 2017

Totani, T., Yoshii, Y., Iwamuro, F., Maihara, T., \& Motohara, K. 2001, ApJ, 558, L87

van Dokkum, P. G., et al. 2003, ApJ, 587, L83

Williams, R. E., et al. 2000, AJ, 120, 2735 